\title{
Phonon-Induced Optical Superlattice
}

\author{
M. M. de Lima, Jr., ${ }^{1, *}$ R. Hey, ${ }^{1}$ P. V. Santos, ${ }^{1}$ and A. Cantarero ${ }^{2}$ \\ ${ }^{1}$ Paul-Drude-Institut für Festkörperelektronik, Hausvogteiplatz, 5-7, 10117 Berlin, Germany \\ ${ }^{2}$ Materials Science Institute, University of Valencia, P.O. Box 22085, E-46071 Valencia, Spain
}

(Received 11 June 2004; published 1 April 2005)

\begin{abstract}
We demonstrate the formation of a dynamic optical superlattice through the modulation of a semiconductor microcavity by stimulated acoustic phonons. The high coherent phonon population produces a folded optical dispersion relation with well-defined energy gaps and renormalized energy levels, which are accessed using reflection and diffraction experiments.
\end{abstract}

PACS numbers: 73.21.Cd, 42.65.Es, 42.70.Qs, 77.65.Dq

The notion of a superlattice, a periodic stack of layers of different materials, was originally introduced as a way of tailoring the electronic band structure of semiconductors via quantum size effects [1]. The dispersion relation of elementary excitations in superlattices is characterized by minibands within a folded mini-Brillouin zone (MBZ) defined by the artificial periodicity and separated by minigaps corresponding to regions without propagating modes. The superlattice concept was later extended to phonons [2]. Although optical superlattices in the form of Bragg mirrors have been known for a long time, their two- and threedimensional counterparts, typically referred to as photonic crystals, are presently receiving considerable attention due to the ability to induce light localization and control spontaneous emission $[3,4]$.

Elementary excitations such as phonons, plasmons, and spin waves also create, when stimulated with a welldefined wave vector, a periodic modulation of the material's optical properties. In fact, this modulation provides a primary source of information about their energy dispersion when accessed using techniques such as Raman and Brillouin spectroscopy. An interesting question is whether an elementary excitation can create a dynamic optical superlattice with a folded dispersion and energy gaps. The interaction with photons, however, is usually very weak with induced energy shifts smaller than the characteristic width of the spectral lines. This interaction becomes enhanced for photon energies in resonance with the elementary excitation (resonant scattering) or by artificially increasing their population above the equilibrium (stimulated scattering). We point out that excitations of a bosonic nature are more appropriate for optical modulation with a high excitation density in order to avoid the broadening of the spectral features due to many-body effects [5]. The previous conditions may lead to the formation of new quasiparticles, as exemplified by the different forms of polaritons.

The interaction with photons can be further enhanced in low-dimensional structures. Strong optical fields in semiconductor microcavities have been shown to increase the cross section for Raman scattering by thermal vibrations by several orders of magnitude [6]. In addition, Brillouin scattering efficiencies exceeding $40 \%$ have been reported in microcavities exposed to a high, nonthermal population of coherent acoustic modes [7]. A zone folded energy dispersion with minigaps has also been predicted for cavity polaritons modulated by coherent acoustic phonons [5]. To our knowledge, however, the formation of a dynamic optical superlattice with a well-defined folded dispersion and stop bands by an elementary excitation has not been reported.

In this Letter, we demonstrate the realization of an optical superlattice through the interaction between photons mediated by stimulated acoustic phonons in a semiconductor microcavity. This interaction leads to new quasiparticles - phonon dressed photons - with an energy dispersion folded within a MBZ defined by the phonon wave vector. The formation of a folded superlattice dispersion is demonstrated by the observation of well-defined modes bounding the stop bands. Furthermore, the microcavity resonance energy is renormalized through the interaction of the photons with phonons in second order perturbation theory. The latter is analogous to the phonon-induced renormalization of the electronic band gap, which accounts for the temperature dependence of most electronic properties. Finally, we show that the dynamic superlattice concept can be extended to two dimen-

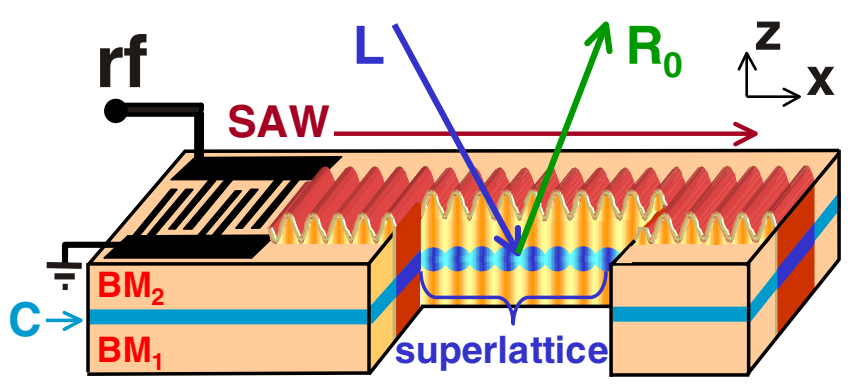

FIG. 1 (color online). Schematic diagram of the optical resonators consisting of a GaAs cavity (C) sandwiched between Bragg mirrors $\left(\mathrm{BM}_{1}\right.$ and $\left.\mathrm{BM}_{2}\right)$ and modulated by a $\mathrm{SAW}$ generated by an interdigital transducer. $\mathrm{L}$ and $\mathrm{R}_{0}$ represent the incident and reflected light beams, respectively. 
sions by interfering acoustic beams propagating along orthogonal directions.

The microcavities investigated here (Fig. 1) consist of a GaAs $\lambda / 2$ optical cavity (C) surrounded by GaAs/AlAs Bragg mirrors $\left(\mathrm{BM}_{1}\right.$ and $\left.\mathrm{BM}_{2}\right)$ grown on (001) GaAs by molecular-beam epitaxy. The layer structure (described in detail in Ref. [7]) is modulated by coherent acoustic phonons in the form of Rayleigh surface acoustic waves (SAWs) generated by an interdigital transducer (IDT) oriented along the $x=[110]$ surface direction. The microcavities display a sharp reflectivity minimum (quality factor $Q=1200$ ) within the stop band defined by the BMs when the vertical (i.e., along $z$ ) component of the wave vector $k_{L}$ of the incident light equals $k_{z}=\pi / d_{\text {eff }}$. Here, $d_{\text {eff }}=n_{c} d_{c}$ denotes the optical thickness of the cavity layer with refractive index $n_{c}$ and physical thickness $d_{c}$. The SAW periodically changes the optical resonance wavelength $\lambda$ according to $\Delta \lambda / \lambda=\Delta d_{\text {eff }} / d_{\text {eff }}=$ $\Delta n_{c} / n_{c}+s_{z z}$, the dominating mechanism being the modulation of the layer thicknesses through the vertical component $s_{z z}$ of the SAW strain field $[7,8]$.

The dynamic superlattice with period $\lambda_{\mathrm{SAW}}$ is formed through the modulation of the optical resonance along $x$. Its photon dispersion as a function of the $x$ component of the light wave vector $\left(k_{x}\right)$ was calculated following the procedure described in Ref. [9]. The calculations are based on the solution of Maxwell's equation using a transfer matrix (TM) method. They take into account the acoustooptic modulation of the refractive index and the thickness of each layer by the SAW field but neglect retardation and electro-optic effects. The thin solid curve in Fig. 2 shows the dispersion for a vanishing SAW intensity and corresponds to the folding into the MBZ defined by

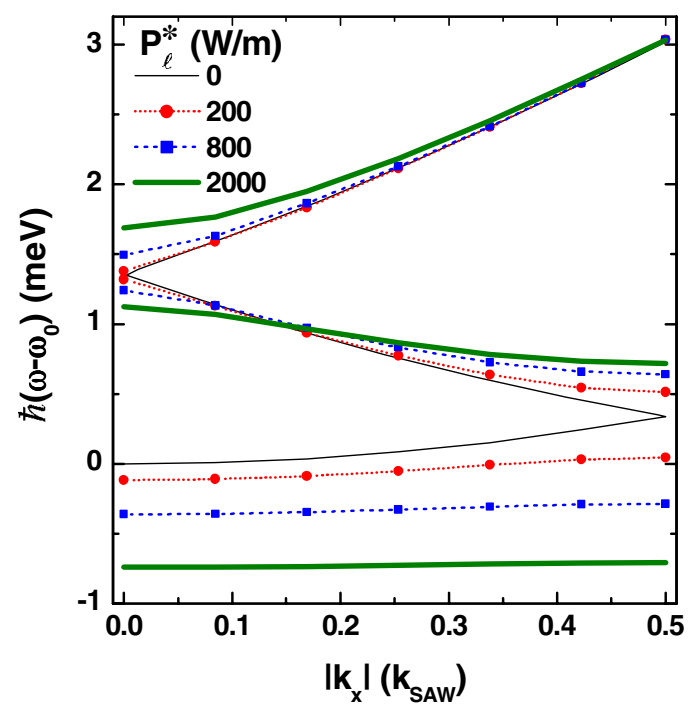

FIG. 2 (color online). Calculated light dispersion of the phonon-induced superlattice for $\lambda_{\mathrm{SAW}}=5.6 \mu \mathrm{m}$ and different acoustic power densities $P_{\ell}^{*}$. The thin solid curve indicates the dispersion for $P_{\ell}^{*} \rightarrow 0$. The energy scales are relative to the cavity resonance energy $\hbar \omega_{0}=1.3535 \mathrm{eV}$.
$\left|k_{x}\right|<k_{\mathrm{SAW}} / 2$ of the quadratic photon dispersion $\hbar \omega=$ $\hbar \omega_{0} \sqrt{1+\left[k_{x} /\left(n_{c} k_{z}\right)\right]^{2}}$ of a planar microcavity. Here, $\hbar \omega_{0}$ denotes the cavity resonance energy for normal incidence and corresponds to the onset of the dispersion in the absence of a SAW. The additional curves were calculated for different effective linear acoustic power densities $\left(P_{\ell}^{*}\right)$, defined as the acoustic power flow per unit length perpendicular to the propagation direction. As $P_{\ell}^{*}$ increases, gaps open up in the center $\left(k_{x}=0\right)$ and at the boundary $\left(\left|k_{x}\right|=\right.$ $\left.k_{\mathrm{SAW}} / 2\right)$ of the MBZ. In addition, the lowest dispersion branch redshifts and becomes essentially flat for high acoustic amplitudes.

The dispersion modes for $k_{x}=0$ were probed by reflectivity measurements under normal incidence $\left(R_{0}\right)$. The incident light was focused onto a spot of diameter $\phi_{d}=$ $40 \mu \mathrm{m}$ in order to probe several SAW periods. The IDTs were powered using a radio frequency (rf) amplifier capable of delivering up to $P_{\mathrm{rf}}=25 \mathrm{dBm}$ at the resonance frequency of $f_{\mathrm{SAW}}=540 \mathrm{MHz}$. The measured linear power density $\left(P_{\ell}\right)$ was determined from $P_{\mathrm{rf}}$ by taking into account the rf coupling losses measured using a spectrum analyzer. All measurements were carried out at room temperature using a modulation technique to correct for eventual temperature-induced energy shifts due to sample heating by the high rf intensities.

Figure 3(a) shows reflectivity spectra recorded under SAWs with wavelengths $\lambda_{\mathrm{SAW}}=5.6$ and $2.8 \mu \mathrm{m}$ and different acoustic power densities. The energy scale is relative to the cavity resonance $\hbar \omega_{0}=1.3535 \mathrm{eV}$ in the absence of a SAW (thin line). The SAW redshifts the cavity resonance and induces new reflection minima above $\hbar \omega_{0}$, which are attributed to the coupling of the incident light to folded modes at the center of the MBZ. This interpretation is corroborated by the blueshift of the high energy minima as the dimensions of the MBZ increase with decreasing $\lambda_{\text {SAW }}$.

In order to analyze further details of the spectra for $\lambda_{\mathrm{SAW}}=5.6 \mu \mathrm{m}$ and $P_{\ell}=500 \mathrm{~W} / \mathrm{m}$ in Fig. 3, we indicate by arrows the positions of the zone center [Fig. 3(a)] and zone edge [Fig. 3(b)] boundary modes calculated by the TM model for an effective $P_{\ell}^{*}=2000 \mathrm{~W} / \mathrm{m}$. We found that it is necessary to use $P_{\ell}^{*}=4 P_{\ell}$ (corresponding to a scaling factor of 2 of the acoustic amplitudes) in order to achieve quantitative agreement between the calculations and the experiments, thus indicating that the effective modulation is larger than the one predicted. This discrepancy is attributed to the approximations used in the TM model or to a possible underestimation of elasto-optical coefficients for the acoustic power levels used here. This behavior, which has also been recently reported for $\mathrm{AlGaN}$ films [10], is presently under investigation. We note, however, that it does not undermine the experimental observation of the modes bounding the minigaps in the dispersion of the optical superlattice.

The minimum at $\hbar\left(\omega-\omega_{0}\right) \approx 2 \mathrm{meV}$ in Fig. 3(a) is assigned to the excitation of the third zone center mode in 


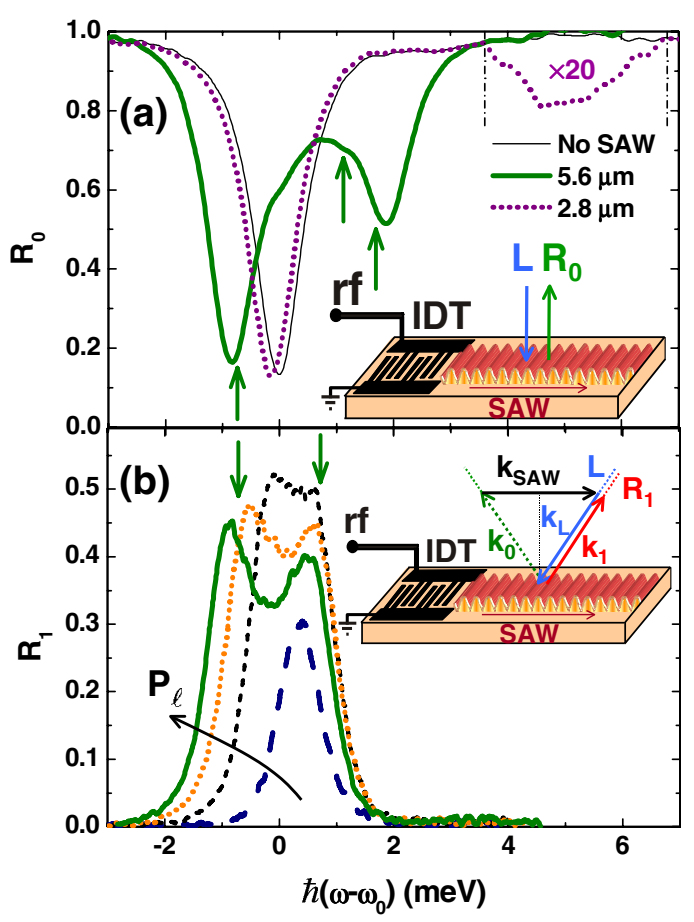

FIG. 3 (color online). (a) Normal incidence reflectivity $\left(R_{0}\right)$ of the cavity structures in the absence of a SAW (thin line) and under SAWs with wavelengths $\lambda_{\mathrm{SAW}}=5.6\left(P_{\ell}=500 \mathrm{~W} / \mathrm{m}\right.$, thick line) and $2.8 \mu \mathrm{m}$ (dotted line). (b) First order diffraction intensity $R_{1}$ from a dynamic superlattice induced by SAWs with $\lambda_{\mathrm{SAW}}=5.6 \mu \mathrm{m}$ and $P_{\ell}=24,160,310$, and $500 \mathrm{~W} / \mathrm{m}$. The experimental configurations are shown in the insets, where $L$ indicates the incoming light beam with wave vector $k_{L}$ while $k_{0}$ and $k_{1}$ denote the wave vectors of the reflected and diffracted modes, respectively. $k_{\mathrm{SAW}}$ represents the acoustic wave vector. The arrows in (a) and (b) indicate the energies of the modes for $k_{x}=0$ and $k_{x}=k_{\mathrm{SAW}} / 2$, respectively, calculated for $\lambda_{\mathrm{SAW}}=$ $5.6 \mu \mathrm{m}$ and $P_{\ell}^{*}=2000 \mathrm{~W} / \mathrm{m}$.

Fig. 2. The second zone center mode, in contrast, causes only a very small reflectivity bump. The large difference in reflectivity for the two modes arises from the different phases $\chi_{x}$ between the light electric field and the SAWinduced modulation of the optical thickness along $x$ $[11,12]$. The latter are in phase (i.e., $\chi_{x}=0$ ) for the third mode, thus ensuring a strong coupling to the incident light beam. For the second mode, $\chi_{x}=90^{\circ}$ and the coupling vanishes.

The modes bounding the first stop band at the edge of the MBZ were accessed by illuminating the samples at the Bragg angle $\theta_{B}=\tan ^{-1}\left[k_{\mathrm{SAW}} /\left(2 k_{0}\right)\right]$ using the configuration displayed in the inset of Fig. 3(b) and measuring the intensity of the backscattered first order diffraction beam $\left(R_{1}\right)$. Results for different acoustic powers are summarized in Fig. 3(b). The spectra for $P_{\ell}<100 \mathrm{~W} / \mathrm{m}$ are characterized by a single line, which appears only when the SAW is switched on. The stop band width under these conditions is narrower than the characteristic width of the spectral lines. For $P_{\ell}>100 \mathrm{~W} / \mathrm{m}$, two lines appear with a splitting $\Delta \hbar \omega_{1}$ proportional to the square root of $P_{\ell}$ [filled squares in Fig. 4(a)]. The two lines are attributed to the excitation of the two phonon dressed photon modes bounding the stop band at the edge of the MBZ. Their positions for $P_{\ell}=$ $500 \mathrm{~W} / \mathrm{m}$ (thick line) agree well with the energies of the boundary modes calculated using the TM model (arrows).

The observation of a folded dispersion with well-defined modes bounding the stop bands clearly demonstrates a strong photon-photon interaction under high acoustic excitation in the dynamic superlattice. We note that other than in a phonon polariton, the phonons in the present case are not resonant with the photons. They act rather as mediators of the coupling between resonant photons in a situation analogous to the role played by phonons in the electron-electron coupling leading to BCS superconductivity.

The dynamic character of the optical superlattice provides the unique opportunity of following the formation of the stop bands with increasing SAW power. We concentrate here on the high symmetry photon states $\left|l_{j}^{\prime}\right\rangle$ in the center ( $j$ even) and at the boundary ( $j$ odd) of the MBZ, which can be written as a superposition of plane waves $\left|l_{j}\right\rangle$ with $k_{x}=j k_{\mathrm{SAW}} / 2$. The energy splitting leading to the stop bands arises from the first order coupling between the $\left|l_{ \pm j}\right\rangle, j \neq 0$ modes with wave vectors differing by a multiple of $k_{\mathrm{SAW}}$. If the acoustic modulation of the BMs is neglected, the photon-phonon interaction Hamiltonian can be written as $\Delta E_{c}=-\hbar \omega_{0} \Delta d_{\text {eff }} / d_{\text {eff }} \cos \left(k_{\mathrm{SAW}} x\right)$ and the ratio between the width $\hbar \Delta \omega_{1}$ and the average energy gap $\hbar \omega_{1}$ of the first zone boundary gap becomes $\Delta \omega_{1} / \omega_{1} \approx$ $\Delta d_{\text {eff }} / d_{\text {eff }} \quad$ [12]. Using the $\Delta d_{\text {eff }} / d_{\text {eff }}=0.55 \times$ $10^{-3} \sqrt{P_{\ell} / P_{\ell, 0}}$ with $P_{\ell, 0}=200 \mathrm{~W} / \mathrm{m}$ determined from

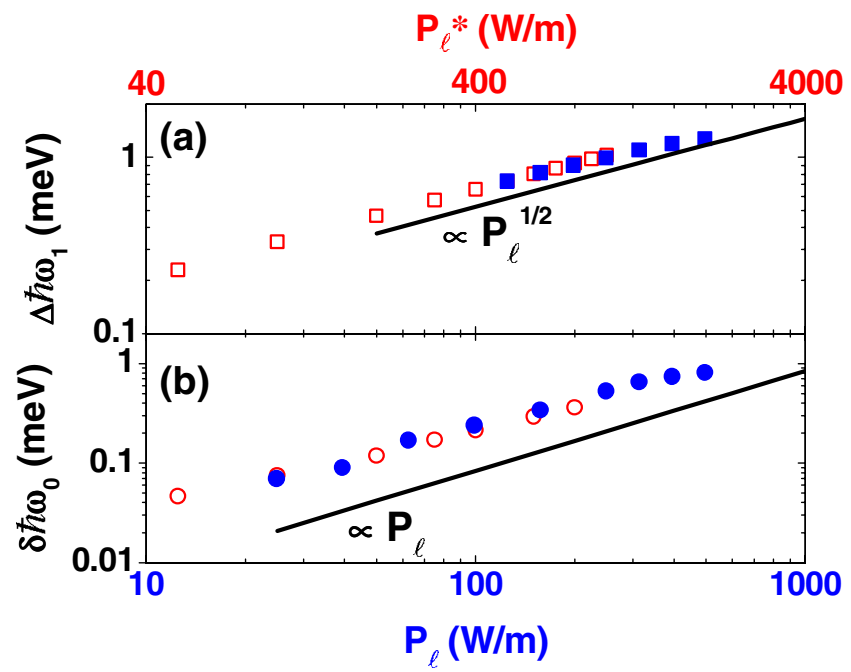

FIG. 4 (color online). (a) Width $\Delta \hbar \omega_{1}$ of the first zone boundary stop band (squares) and (b) redshift $\delta \hbar \omega_{0}$ of the cavity resonance (dots) as a function of the acoustic power density. Open symbols (upper scale) and filled symbols (lower scale) are calculated and measured values, respectively. The solid lines represent the expected behavior using the analytical model described in the text. 
the acoustic properties of the sample, the previous analytical expression yields a stop band width approximately $20 \%$ smaller than the measured ones [solid line in Fig. 4(a)]. The open squares in Fig. 4(a) display the predictions of the TM model, which takes into account the depth dependence of the acoustic fields across the structure.

A further interesting aspect of the acoustic modulation is the redshift $\delta \hbar \omega_{0}$ of the cavity resonance with increasing $P_{\ell}$ [filled dots in Fig. 4(b)]. This redshift, which is analogous to the temperature-induced renormalization of the electronic band gap [13], can be expressed in the harmonic approximation as

$$
\delta \hbar \omega_{0} \approx \underbrace{\left\langle l_{0}\left|\Delta \mathbf{E}_{\mathbf{c}}(\mathbf{u})\right| l_{0}\right\rangle}_{\delta E_{D W}}+\underbrace{\sum_{j= \pm 2} \frac{\left|\left\langle l_{0}\left|\Delta \mathbf{E}_{\mathbf{c}}(\mathbf{u})\right| l_{j}\right\rangle\right|^{2}}{E_{l_{0}}-E_{l_{j}}}}_{\delta E_{\mathrm{SE}}},
$$

where $\mathbf{u}$ represents the phonon amplitude. Equation (1) neglects the contribution from the phonon-induced lattice expansion, which can be shown to be small for the present case. The first correction $\delta E_{\mathrm{DW}}$ arises from the nonlinear dependence of the optical thickness on the amplitude of the acoustic fields, leading to a nonvanishing value for $\Delta d_{\text {eff }}$ when averaged over a SAW period. This term is analogous to the Debye-Waller contribution to the renormalization of the electronic band gap and can be approximated by $\delta E_{\mathrm{DW}} \approx \hbar \omega_{0}\left(\Delta d_{\mathrm{eff}} / d_{\mathrm{eff}}\right)^{2}$. This term was also found to be small compared to the measured values in Fig. 4(b). The most important contribution comes from $\delta E_{\mathrm{SE}}$, which is a self-energy correction induced by the emission and subsequent absorption of a SAW quantum. Using the approximations described above, one obtains $\delta E_{\mathrm{SE}}=$ $\frac{\left(\hbar \omega_{0}\right)^{2}}{2\left[\hbar \omega_{0}-\hbar \omega_{2}\right]}\left[\Delta d_{\text {eff }} / d_{\text {eff }}\right]^{2} \propto P_{\ell}$. This approximation [solid line in Fig. 4(b)] reproduces the observed linear dependence of $\delta \omega_{0}$ on $P_{\ell}$, as well as the energy renormalization obtained from the TM method (open circles).

The dynamic superlattice concept can be extended to two-dimensional photonic lattices by interfering orthogonal SAW beams to form a square lattice, as indicated in the inset of Fig. 5. The reciprocal lattice is generated by primitive vectors $\mathbf{k}_{10}$ and $\mathbf{k}_{11}$ with different lengths $k_{10}=$ $2 \pi / \lambda_{\text {SAW }}$ and $k_{11}=\sqrt{2} k_{10}$, respectively. The latter leads to different Bragg angles along the [1 0$]$ and the [1 1 ] directions, as shown in the measurements of the first order diffracted beam $R_{1}$ in Fig. 5 .

In conclusion, we have demonstrated the formation of a dynamic optical superlattice through the modulation of a semiconductor resonator by stimulated acoustic phonons. The high phonon population leads to an energy renormalization and folding of the original photon dispersion within a MBZ defined by the phonon wave vector. The folded dispersion has well-defined stop bands. Finally, the concepts can be extended to two dimensions, thus providing a way to realize tunable photonic crystals.

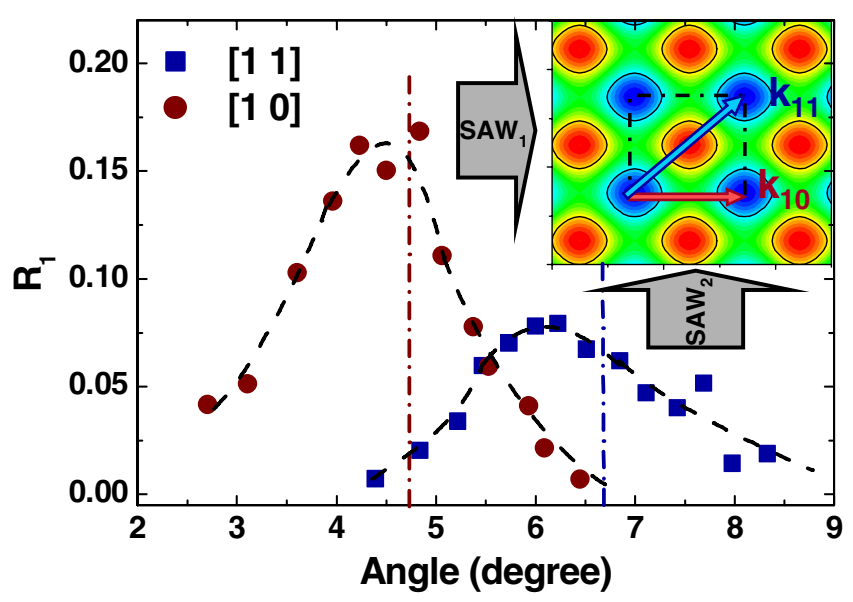

FIG. 5 (color online). Inset: Two-dimensional square superlattice with primitive wave vectors $k_{10}$ and $k_{11}$ formed by the interference of two orthogonal SAW beams. Main plot: The diffracted intensity $R_{1}$ as a function of the incidence angle for the incidence plane parallel to the [1 0$]$ (circles) and to the [ 11 1 (squares) directions. The dash-dotted lines indicate the corresponding Bragg angles.

We thank K.-J. Friedland and J. A. H. Stotz for comments and for a critical reading of the manuscript, as well as M. Höricke for MBE growth and S. Krauß and W. Seidel for the preparation of the samples. Support from the Deutsche Forschungsgemeinschaft and from the DAAD (Germany)/Acciones Integradas (Spain) is gratefully acknowledged.

*Electronic address: mmlimajr@pdi-berlin.de

[1] L. Esaki and R. Tsu, IBM J. Res. Dev. 14, 61 (1970).

[2] C. Colvard, R. Merlin, M. V. Klein, and A. C. Gossard, Phys. Rev. Lett. 45, 298 (1980).

[3] S. John, Phys. Rev. Lett. 58, 2486 (1987).

[4] E. Yablonovitch, Phys. Rev. Lett. 58, 2059 (1987).

[5] A. L. Ivanov and P. B. Littlewood, Phys. Rev. Lett. 87, 136403 (2001).

[6] A. Fainstein, B. Jusserand, and V. Thierry-Mieg, Phys. Rev. Lett. 75, 3764 (1995).

[7] M. M. de Lima, Jr., R. Hey, and P. V. Santos, Appl. Phys. Lett. 83, 2997 (2003).

[8] M. M. de Lima, Jr., P. V. Santos, R. Hey, and S. Krishnamurthy, Physica (Amsterdam) 21E, 809 (2004).

[9] S. Krishnamurthy and P. V. Santos, J. Appl. Phys. 96, 1803 (2004).

[10] G. Bu et al., Appl. Phys. Lett. 85, 2157 (2004).

[11] B. Jusserand, F. Alexandre, J. Dubard, and D. Paquet, Phys. Rev. B 33, R2897 (1986).

[12] P. V. Santos, L. Ley, J. Mebert, and O. Koblinger, Phys. Rev. B 36, 4858 (1987).

[13] P. Allen and V. Heine, J. Phys. C 9, 2305 (1976). 